\title{
Tails of random sums of a heavy-tailed number of light-tailed terms *
}

\author{
Christian Y. Robert ${ }^{\text {a,* }}$ \\ ${ }^{a}$ ENSAE, Timbre J120, 3 Avenue Pierre Larousse, 92245 MALAKOFF Cedex, \\ France \\ Johan Segers ${ }^{b}$ \\ ${ }^{\mathrm{b}}$ Université catholique de Louvain, Institut de statistique, Voie du Roman Pays 20, \\ B-1348 Louvain-la-Neuve, Belgium
}

\begin{abstract}
The tail of the distribution of a sum of a random number of independent and identically distributed nonnegative random variables depends on the tails of the number of terms and of the terms themselves. This situation is of interest in the collective risk model, where the total claim size in a portfolio is the sum of a random number of claims. If the tail of the claim number is heavier than the tail of the claim sizes, then under certain conditions the tail of the total claim size does not change asymptotically if the individual claim sizes are replaced by their expectations. The conditions allow the claim number distribution to be of consistent variation or to be in the domain of attraction of a Gumbel distribution with a mean excess function that grows to infinity sufficiently fast. Moreover, the claim number is not necessarily required to be independent of the claim sizes.
\end{abstract}

Key words: Compound distribution; Collective risk model; Consistent variation; Heavy-tailed distributions; Light-tailed distributions; Large deviations; Random sum.

ऋ Research supported financially by the AXA chair "Large Risks in Insurance" (C. Y. Robert) and by the IAP research network grant nr. P6/03 of the Belgian government (J. Segers). The authors gratefully acknowledge an anonymous referee for various suggestions leading to improved results throughout the paper.

* Corresponding author.

Email addresses: chrobert@ensae.fr (Christian Y. Robert), Johan. Segers@uclouvain. be (Johan Segers).

Preprint submitted to Insurance: Mathematics and Economics 2 February 2008 


\section{Introduction}

In the collective risk model in actuarial risk theory, the total claim amount in a portfolio is assumed to be a random sum,

$$
S_{N}=\sum_{i=1}^{N} X_{i}
$$

where $N$, the claim number, is a nonnegative integer-valued random variable and $X_{1}, X_{2}, \ldots$, the claim sizes, form a sequence of independent and identically distributed (iid) nonnegative random variables, independent of $N$; see e.g. Kaas et al. (2001, chapter 3). The tail of the compound distribution depends on the tails of the claim number and claim size distributions. If both distributions are light-tailed, that is, if $\mathrm{E}\left[e^{t N}\right]<\infty$ and $\mathrm{E}\left[e^{t X_{1}}\right]<\infty$ for some $t>0$, saddlepoint approximation techniques can be used to analyze the tail of $S_{N}$ (Beard et al., 1984; Jensen, 1995). If the individual claim distribution is subexponential and if the claim number distribution is light-tailed, then

$$
\operatorname{Pr}\left[S_{N}>x\right] \sim \mathrm{E}[N] \operatorname{Pr}\left[X_{1}>x\right], \quad x \rightarrow \infty
$$

(Embrechts et al., 1997, Theorem A3.20); see Denisov et al. (2007) for onesided versions of eq. (1.1) under milder conditions.

In this paper we concentrate on the converse case when the tail of $S_{N}$ is dominated by the tail of $N$. This setting is relevant, for instance, for earthquake insurance, featuring a potentially large number of bounded claims, or in the case of individual unobserved heterogeneity, as well as in queueing theory, see Section 6. We try to answer to the following question raised in Schmidli (1999): When does

$$
\operatorname{Pr}\left[S_{N}>x\right] \sim \operatorname{Pr}\left[\mathrm{E}\left[X_{1}\right] N>x\right], \quad x \rightarrow \infty
$$

hold? We consider a slightly more general framework because we do not necessarily assume the claim number to be independent of the claim sizes.

Theorems 1.3 and 1.4 in Stam (1973) shed light on the difference between the two approximations in eqs. (1.1) and (1.2): if the claim number and claim size distributions have finite expectations and regularly varying tails, that is, if there exist $\alpha>1$ and $\beta>1$ such that

$$
\lim _{x \rightarrow \infty} \frac{\operatorname{Pr}[N>x y]}{\operatorname{Pr}[N>x]}=y^{-\alpha}, \quad \lim _{x \rightarrow \infty} \frac{\operatorname{Pr}\left[X_{1}>x y\right]}{\operatorname{Pr}\left[X_{1}>x\right]}=y^{-\beta}
$$

for $y>0$, then, provided $N$ and $\left\{X_{i}\right\}_{i \geq 1}$ are independent, $\alpha>\beta$ implies (1.1) while $\beta>\alpha$ implies (1.2). In other words, which of the two approximations (1.1) or (1.2) is valid depends on which of the two distributions has the heavier tail. Approximation (1.2) will therefore arise when the tail of $N$ is heavier than the tail of $X$. 
The main contribution of this paper consists of the four theorems in Sections 3 and 4 providing different sets of sufficient conditions for eq. (1.2) to hold. The two theorems in Section 3 concern the case where the claim number distribution is of consistent variation and the claim size distribution has a finite moment of order $r$ for some $r>1$. The two theorems in Section 4 treat the case where the claim number distribution is in the Gumbel domain of attraction and the moment generating function of the claim size distribution is finite in a neighbourhood of the origin. An important factor is the speed at which the mean excess function $\mathrm{E}[N-x \mid N>x]$ tends to infinity. In the special case where $N$ is a discretized Weibull random variable with tail function $\operatorname{Pr}[N>x] \sim \exp \left(-x^{\beta}\right)$ as $x \rightarrow \infty$ and shape parameter $0<\beta<1$, the conditions for Theorems 4.1 and 4.2 translate into $\beta<1 / 3$ and $\beta<1 / 2$, respectively. The exponent $1 / 2$ marks the lower boundary on the speed of growth of the mean excess function for which eq. (1.2) can still be expected to hold; see Section 5. In Theorems 3.1 and 4.1, the dependence between the claim number and the claim sizes can be arbitrary.

The outline of this paper is as follows: Section 2 presents preliminaries on tail asymptotics and large deviations. Statements and proofs of our main results are to be found in Sections 3 and 4. Section 5 gives a short discussion. Section 6 concludes the paper with applications to insurance and operations research.

The following notations and assumptions are in force throughout the paper. Let $N$ be a nonnegative integer-valued random variable and let $X_{1}, X_{2}, \ldots$ be a sequence of iid nonnegative random variables with finite mean $\mu=\mathrm{E}\left[X_{1}\right]$ and variance $\sigma^{2}=\operatorname{Var}\left[X_{1}\right]$. The claim number $N$ is not necessarily assumed to be independent of $\left\{X_{i}\right\}_{i \geq 1}$. Denote $S_{0}=0$ and $S_{n}=X_{1}+\cdots+X_{n}$ for an integer $n \geq 1$. For real $y$, we denote by $\lceil y\rceil$ the smallest integer greater than or equal to $y$; similarly, $\lfloor y\rfloor$ is the greatest integer smaller than or equal to $y$. For two positive functions $f$ and $g$ defined in a neighbourhood of infinity, we write $f(x) \sim g(x)$ as $x \rightarrow \infty$ provided $\lim _{x \rightarrow \infty} f(x) / g(x)=1$ and we write $f(x)=o(g(x))$ as $x \rightarrow \infty$ provided $\lim _{x \rightarrow \infty} f(x) / g(x)=0$. The indicator function of an event $A$ is denoted by $\mathbf{1}(A)$.

\section{Preliminaries}

\subsection{Consistent variation}

A distribution function $F$ is said to have a consistently varying tail, notation $F \in \mathcal{C}$, if

$$
\lim _{y \uparrow 1} \limsup _{x \rightarrow \infty} \frac{\bar{F}(x y)}{\bar{F}(x)}=1,
$$


where $\bar{F}=1-F$. This regularity property was first introduced in Cline (1994) and called "intermediate regular variation". The concept of consistent variation has been proven useful in various papers in queueing systems and ruin theory (Cai and Tang, 2004; Kaas and Tang, 2005; Ng et al., 2004; Tang, 2004).

A distribution function $F$ is said to have a regularly varying tail, notation $F \in \mathcal{R}$, if there exists $\alpha>0$ such that

$$
\lim _{x \rightarrow \infty} \frac{\bar{F}(x y)}{\bar{F}(x)}=y^{-\alpha}, \quad y>0,
$$

or equivalently such that

$$
\bar{F}(x)=x^{-\alpha} L(x), \quad x>0,
$$

where $L$ is a slowly varying function, that is, a function satisfying

$$
\lim _{x \rightarrow \infty} \frac{L(x y)}{L(x)}=1, \quad y>0 .
$$

In Cline and Samorodnitsky (1994), it is shown that the class $\mathcal{C}$ strictly contains the class $\mathcal{R}$. In addition the class $\mathcal{C}$ is itself strictly contained in the class of distribution functions with a dominatedly varying tail, that is, the class $\mathcal{D}$ of distribution functions $F$ such that

$$
\limsup _{x \rightarrow \infty} \frac{\bar{F}(x y)}{\bar{F}(x)}<\infty
$$

for all (or, equivalently, for some) $y \in(0,1)$.

For a distribution function $F$, define

$$
\bar{F}_{*}(y)=\liminf _{x \rightarrow \infty} \frac{\bar{F}(x y)}{\bar{F}(x)}, \quad y>0,
$$

and

$$
\alpha_{F}=\lim _{y \rightarrow \infty} \frac{-\log \left\{\bar{F}_{*}(y)\right\}}{\log y}<\infty,
$$

with $-\log 0=\infty$. In the terminology of Bingham et al. (1987, Proposition 2.2.5), the quantity $\alpha_{F}$ is the upper Matuszewska index of the function $1 / \bar{F}$. It is an upper bound for the upper order, $\rho_{F}$, of $1 / \bar{F}$, defined by

$$
\rho_{F}=\limsup _{x \rightarrow \infty} \frac{-\log \{\bar{F}(x)\}}{\log x} .
$$

By Bingham et al. (1987, Theorem 2.1.7), a distribution function $F$ belongs to $\mathcal{D}$ if and only if $\alpha_{F}<\infty$. But then $\rho_{F} \leq \alpha_{F}<\infty$. As $\bar{F}(x)=x^{-\rho(x)}$ with 
$\lim \sup _{x \rightarrow \infty} \rho(x)=\rho_{F}$, we obtain that for every $\rho>\rho_{F}$,

$$
x^{-\rho}=o(\bar{F}(x)), \quad x \rightarrow \infty .
$$

In this sense, distributions of dominated variation (and thus also those of consistent variation) are heavy-tailed.

\subsection{Domains of attraction}

Let $N$ be a nonnegative integer-valued random variable. Assume that $N$ is in the maximal domain of attraction of an extreme value distribution. Because the distribution of $N$ is discrete, its attractor must be a Fréchet or the Gumbel distribution, that is, its attractor cannot be a Weibull extreme value distribution.

On the one hand, the distribution of $N$ is in the domain of attraction of the Fréchet distribution with shape parameter $\alpha>0$ if and only if

$$
\operatorname{Pr}[N>x]=x^{-\alpha} L(x)
$$

where $L$ is a slowly varying function. This case was treated in Stam (1973) and in Fay et al. (2006); see Section 6. A sufficient condition for (2.5) is the von Mises type condition $\lim _{n \rightarrow \infty} n \operatorname{Pr}[N=n] / \operatorname{Pr}[N>n]=\alpha$ (Anderson, 1980). In turn, regular variation implies consistent variation, that is, (2.5) implies (2.1) with $F(x)=\operatorname{Pr}[N \leq x]$, and it is merely the latter concept which will be used later on.

On the other hand, the distribution of $N$ is in the domain of attraction of the Gumbel distribution if and only if there exists a positive function $a$ defined in a neighbourhood of infinity such that

$$
\lim _{x \rightarrow \infty} \frac{\operatorname{Pr}[N>x+y a(x)]}{\operatorname{Pr}[N>x]}=e^{-y}, \quad y \in \mathbb{R} .
$$

The function $a$ is necessarily asymptotically equivalent to the mean excess function, $a(x) \sim \mathrm{E}[N-x \mid N>x]$ as $x \rightarrow \infty$, and it is self-neglecting, that is,

$$
\lim _{x \rightarrow \infty} \frac{a(x)}{x}=0, \quad \lim _{x \rightarrow \infty} \frac{a(x+y a(x))}{a(x)}=1, \quad y \in \mathbb{R} .
$$

The tail function of $N$ admits the following useful representation:

$$
\operatorname{Pr}[N>x]=c(x) \exp \left(-\int_{0}^{x} \frac{g(t)}{a(t)} d t\right)
$$


where $\lim _{x \rightarrow \infty} c(x)=c \in(0, \infty)$ and $\lim _{t \rightarrow \infty} g(t)=1$. By Anderson (1980), a sufficient condition for $N$ to be in the Gumbel domain of attraction is that $q_{n} \rightarrow \infty$ and $q_{n+1}-q_{n} \rightarrow 0$ as $n \rightarrow \infty$, where $q_{n}=\operatorname{Pr}[N>n] / \operatorname{Pr}[N=n]$.

Example. Let $N$ be a discretized Weibull variable, that is, $N=\lfloor Y\rfloor$ where $\operatorname{Pr}[Y>y]=\exp \left(-y^{\beta}\right)$ for $y \geq 0$, with parameter $\beta>0$. If $\beta<1$, then $\operatorname{Pr}[N>y] \sim \operatorname{Pr}[Y>y]$ as $y \rightarrow \infty$ and (2.6)-(2.8) hold with $a(x)=\beta^{-1} x^{1-\beta}$.

\subsection{Large deviations}

In order to analyse the tails of the compound sum $S_{N}$, we need bounds on the probability of large deviations of $S_{n}$. The first bound is a special case of Lemma 2.3 in Tang (2006).

Lemma 2.1 If $\mathrm{E}\left[X_{1}^{r}\right]<\infty$ for some $r>1$, then for each $\gamma>0$ and $q>0$, there exist positive numbers $v$ and $C=C(v, \gamma)$ irrespective to $x$ and $n$ such that for all $x \geq \gamma n$ and $n=1,2, \ldots$,

$$
\operatorname{Pr}\left[S_{n}-n \mu>x\right] \leq n \operatorname{Pr}\left[X_{1}-\mu>v x\right]+C x^{-q}
$$

The second result is a simple consequence of Cramér's theorem on large deviations; see Feller (1971, equation (7.30), p. 553) or Baltrunas et al. (2004, Lemma 2.2).

Lemma 2.2 If $\mathrm{E}\left[e^{t X_{1}}\right]<\infty$ for some $t>0$, then for any sequence $a_{n}$ satisfying $a_{n} / n^{1 / 2} \rightarrow \infty$ and $a_{n} / n \rightarrow 0$ as $n \rightarrow \infty$,

$$
\begin{aligned}
& \operatorname{Pr}\left[S_{n}>n \mu+a_{n}\right]=\exp \left(-\frac{1}{2 \sigma^{2}} \frac{a_{n}^{2}}{n}\{1+o(1)\}\right) \\
& \operatorname{Pr}\left[S_{n}<n \mu-a_{n}\right]=\exp \left(-\frac{1}{2 \sigma^{2}} \frac{a_{n}^{2}}{n}\{1+o(1)\}\right) .
\end{aligned}
$$

\section{Main results: Consistent variation}

In this section, we treat the case where the tail of the distribution function of $N$ is of consistent variation, see eq. (2.1). Theorem 3.1 states a simple sufficient condition on the common distribution of the $X_{i}$ for eq. (1.2) to be valid for arbitrary dependence structure between $N$ and $\left\{X_{i}\right\}$. In case of independence, a weaker condition suffices, see Theorem 3.2. The results crucially rest on a 
large-deviations result by Tang (2006, Lemma 2.3), reproduced as Lemma 2.1 above, and which was kindly pointed out to us by an anonymous Referee.

Theorem 3.1 If the tail of the distribution of $N$ is of consistent variation, if $\mathrm{E}\left[X_{1}^{r}\right]<\infty$ for some $r>1$ and if

$$
x \operatorname{Pr}\left[X_{1}>x\right]=o(\operatorname{Pr}[N>x]), \quad x \rightarrow \infty,
$$

then $\operatorname{Pr}\left[S_{N}>x\right] \sim \operatorname{Pr}[N>x / \mu]$ as $x \rightarrow \infty$.

Proof. Since $\operatorname{Pr}\left[S_{N}>x\right]=\operatorname{Pr}\left[S_{N} / \mu>x / \mu\right]$, we can without loss of generality assume that $\mu=1$. Fix $0<\varepsilon<1$. For $x>0$,

$$
\operatorname{Pr}\left[S_{N}>x\right]=\operatorname{Pr}\left[S_{N}>x, N \leq(1-\varepsilon) x\right]+\operatorname{Pr}\left[S_{N}>x, N>(1-\varepsilon) x\right],
$$

so that, on the one hand,

$$
\operatorname{Pr}\left[S_{N}>x\right] \leq \operatorname{Pr}\left[S_{\lfloor(1-\varepsilon) x\rfloor}>x\right]+\operatorname{Pr}[N>(1-\varepsilon) x],
$$

and, on the other hand,

$$
\begin{aligned}
\operatorname{Pr}\left[S_{N}>x\right] & \geq 0+\operatorname{Pr}\left[S_{\lceil(1+\varepsilon) x\rceil}>x, N>(1+\varepsilon) x\right] \\
& \geq \operatorname{Pr}[N>(1+\varepsilon) x]-\operatorname{Pr}\left[S_{\lceil(1+\varepsilon) x\rceil} \leq x\right] .
\end{aligned}
$$

Since the tail of $N$ is of consistent variation, by eq. (2.1),

$$
\lim _{\varepsilon \downarrow 0} \liminf _{x \rightarrow \infty} \frac{\operatorname{Pr}[N>(1+\varepsilon) x]}{\operatorname{Pr}[N>(1-\varepsilon) x]}=1 .
$$

In view of eqs. (3.3), (3.4) and (3.5), $\operatorname{Pr}\left[S_{N}>x\right] \sim \operatorname{Pr}[N>x]$ as $x \rightarrow \infty$ will follow if we can show that

$$
\begin{array}{ll}
\operatorname{Pr}\left[S_{\lfloor(1-\varepsilon) x\rfloor}>x\right]=o(\operatorname{Pr}[N>x]), & x \rightarrow \infty \\
\operatorname{Pr}\left[S_{\lceil(1+\varepsilon) x\rceil} \leq x\right]=o(\operatorname{Pr}[N>x]), & x \rightarrow \infty
\end{array}
$$

We first show eq. (3.6). Let $\alpha_{N}$ be the upper Matuszewska index of the function $x \mapsto 1 / \operatorname{Pr}[N>x]$. Since the tail of $N$ is of consistent variation, $\alpha_{N}<\infty$ (see subsection 2.1). Pick $\rho>\alpha_{N}$. By eq. (2.3),

$$
x^{-\rho}=o(\operatorname{Pr}[N>x]), \quad x \rightarrow \infty .
$$

By Lemma 2.1, there exist positive numbers $v$ and $C$ such that 


$$
\begin{aligned}
\operatorname{Pr}\left[S_{\lfloor(1-\varepsilon) x\rfloor}>x\right] & \leq \operatorname{Pr}\left[S_{\lfloor(1-\varepsilon) x\rfloor}-\lfloor(1-\varepsilon) x\rfloor>\varepsilon x\right] \\
& \leq\lfloor(1-\varepsilon) x\rfloor \operatorname{Pr}\left[X_{1}>v \varepsilon x\right]+C(\varepsilon x)^{-\rho}
\end{aligned}
$$

and thus

$$
\frac{\operatorname{Pr}\left[S_{\lfloor(1-\varepsilon) x\rfloor}>x\right]}{\operatorname{Pr}[N>x]} \leq \frac{1}{v \varepsilon} \frac{v \varepsilon x \operatorname{Pr}\left[X_{1}>v \varepsilon x\right]}{\operatorname{Pr}[N>v \varepsilon x]} \frac{\operatorname{Pr}[N>v \varepsilon x]}{\operatorname{Pr}[N>x]}+\frac{C(\varepsilon x)^{-\rho}}{\operatorname{Pr}[N>x]}
$$

Eq. (3.6) now follows from the above inequality combined with eqs. (2.2), (3.1), and (3.8).

Next we show eq. (3.7). Since the $X_{i}$ are nonnegative, by Chernoff's bound, there exists $0<a<1$, depending on $\varepsilon>0$, such that for all sufficiently large $x$

$$
\operatorname{Pr}\left[S_{\lceil(1+\varepsilon) x\rceil} \leq x\right] \leq a^{x} .
$$

This inequality in combination with (3.8) yields eq. (3.7).

Theorem 3.2 Assume that $N$ and $\left\{X_{i}\right\}_{i \geq 1}$ are independent. If the tail of the distribution of $N$ is of consistent variation, if $\mathrm{E}\left[X_{1}^{r}\right]<\infty$ for some $r>1$ and if one of the following two conditions holds:

case $\mathrm{E}[N]<\infty$ :

$$
\operatorname{Pr}\left[X_{1}>x\right]=o(\operatorname{Pr}[N>x]), x \rightarrow \infty
$$

case $\mathrm{E}[N]=\infty$ : there exists $q$ with $1 \leq q<r$ such that

$$
\limsup _{x \rightarrow \infty} \frac{\mathrm{E}[N \mathbf{1}(N \leq x)]}{x^{q} \operatorname{Pr}[N>x]}<\infty
$$

then $\operatorname{Pr}\left[S_{N}>x\right] \sim \operatorname{Pr}[N>x / \mu]$ as $x \rightarrow \infty$.

Proof. The proof is similar to that of Theorem 3.1. We just indicate the modifications. In view of eq. (3.2), it suffices to show that

$$
\operatorname{Pr}\left[S_{N}>x, N \leq(1-\varepsilon) x\right]=o(\operatorname{Pr}[N>x]), \quad x \rightarrow \infty .
$$

Since $N$ and $\left\{X_{i}\right\}_{i \geq 1}$ are independent,

$$
\operatorname{Pr}\left[S_{N}>x, N \leq(1-\varepsilon) x\right]=\sum_{k=0}^{\lfloor(1-\varepsilon) x\rfloor} \operatorname{Pr}[N=k] \operatorname{Pr}\left[S_{k}-k>x-k\right] .
$$

Pick $\rho>\alpha_{N}$, the upper Matuszewska index of the function $x \mapsto 1 / \operatorname{Pr}[N>x]$. By Lemma 2.1, there exist positive numbers $v$ and $C$ such that 


$$
\begin{aligned}
& \operatorname{Pr}\left[S_{N}>x, N \leq(1-\varepsilon) x\right] \\
& \leq \sum_{k=0}^{\lfloor(1-\varepsilon) x\rfloor} \operatorname{Pr}[N=k]\left(k \operatorname{Pr}\left[X_{1}>v(x-k)\right]+\frac{C}{(x-k)^{\rho}}\right) \\
& \leq \operatorname{Pr}\left[X_{1}>\varepsilon v x\right] \sum_{k=0}^{\lfloor(1-\varepsilon) x\rfloor} \operatorname{Pr}[N=k] k+\frac{C}{(\varepsilon x)^{\rho}} \\
& =\operatorname{Pr}\left[X_{1}>\varepsilon v x\right] \mathrm{E}[N \mathbf{1}\{N \leq(1-\varepsilon) x\}]+\frac{C}{(\varepsilon x)^{\rho}}
\end{aligned}
$$

In view of eq. (3.8), we only need to deal with the first term on the right-hand side of the previous display. We now invoke the additional condition. On the one hand, if $\mathrm{E}[N]<\infty$, then also $\mathrm{E}[N \mathbf{1}\{N \leq(1-\varepsilon) x\}] \leq \mathrm{E}[N]<\infty$, and

$$
\frac{\operatorname{Pr}\left[X_{1}>\varepsilon v x\right]}{\operatorname{Pr}[N>x]}=\frac{\operatorname{Pr}[N>\varepsilon v x]}{\operatorname{Pr}[N>x]} \frac{\operatorname{Pr}\left[X_{1}>\varepsilon v x\right]}{\operatorname{Pr}[N>\varepsilon v x]} \rightarrow 0, \quad x \rightarrow \infty,
$$

where we used eqs. (2.2) and (3.10). On the other hand, if $\mathrm{E}[N]=\infty$, then, as $\operatorname{Pr}\left[X_{1}>x\right] \leq \mathrm{E}\left[X_{1}^{r}\right] / x^{r}$,

$$
\begin{aligned}
\operatorname{Pr}\left[X_{1}>\varepsilon v x\right] \mathrm{E}[N \mathbf{1}\{N \leq(1-\varepsilon) x\}] & =O\left(x^{-r}\right) O\left(x^{q} \operatorname{Pr}[N>(1-\varepsilon) x]\right) \\
& =o(\operatorname{Pr}[N>x]), \quad x \rightarrow \infty,
\end{aligned}
$$

as required.

Remark. Observe that if $\mathrm{E}[N]=\infty$ and the function $x \mapsto \operatorname{Pr}[N>x]$ is regularly varying of index $-\alpha$ for some $\alpha \in(0,1]$, then eq. (3.11) holds, for, by Karamata's theorem (Bingham et al., 1987, Propositions 1.5.8 and 1.5.9a),

$\mathrm{E}[N \mathbf{1}(N \leq x)] \leq \int_{0}^{x} \operatorname{Pr}[N>y] d y \begin{cases}\sim x \operatorname{Pr}[N>x] /(1-\alpha) & \text { if } 0<\alpha<1 \\ =o\left(x^{q} \operatorname{Pr}[N>x]\right) & \text { if } \alpha=1<q .\end{cases}$

\section{Main results: Gumbel domain of attraction}

Next, we treat the case where the claim number distribution is in the Gumbel domain of attraction (see subsection 2.2) and the moment generating function of the claim size distribution is finite in a neighbourhood of the origin. An important factor is the speed at which the auxiliary function $a$ in (2.6) tends to infinity. If $a(x) / x^{2 / 3} \rightarrow \infty$ as $x \rightarrow \infty$, then (1.2) holds without further conditions (Theorem 4.1). If the function $a$ is merely assumed to have a lower order larger than 1/2 in the terminology of Bingham et al. (1987, Section 2.2.2, p. 73), then (1.2) still holds provided the claim number is independent of the 
claim sizes (Theorem 4.2). The order $1 / 2$ marks the lower boundary on the speed of growth of $a$ for which (1.2) can still be expected to hold true; see Section 5 .

Theorem 4.1 If $\mathrm{E}\left[e^{\gamma X_{1}}\right]<\infty$ for some $\gamma>0$ and if (2.6) holds for a function a such that

$$
\frac{a(x)}{x^{2 / 3}} \rightarrow \infty, \quad x \rightarrow \infty
$$

then $\operatorname{Pr}\left[S_{N}>x\right] \sim \operatorname{Pr}[N>x / \mu]$ as $x \rightarrow \infty$.

Proof. Without loss of generality, assume $\mu=1$. Fix $\varepsilon>0$. Write

$$
\operatorname{Pr}\left[S_{N}>x\right]=\operatorname{Pr}\left[S_{N}>x, N \leq x-\varepsilon a(x)\right]+\operatorname{Pr}\left[S_{N}>x, N>x-\varepsilon a(x)\right] .
$$

By Lemma 2.2, the first term on the right-hand side of (4.2) is bounded by

$$
\begin{aligned}
\operatorname{Pr}\left[S_{N}>x, N \leq x-\varepsilon a(x)\right] & \leq \operatorname{Pr}\left[S_{\lfloor x-\varepsilon a(x)\rfloor}>x\right] \\
& =\exp \left(-\frac{\varepsilon^{2}}{2 \sigma^{2}} \frac{a^{2}(x)}{x}\{1+o(1)\}\right), \quad x \rightarrow \infty .
\end{aligned}
$$

By (4.1), $a^{2}(x) / x$ is of larger order than $\int_{0}^{x} a^{-1}(t) d t$, so that the right-hand side in the previous display must be $o(\operatorname{Pr}[N>x])$ as $x \rightarrow \infty$.

The second term on the right-hand side of (4.2) is bounded from above by

$$
\operatorname{Pr}\left[S_{N}>x, N>x-\varepsilon a(x)\right] \leq \operatorname{Pr}[N>x-\varepsilon a(x)]
$$

and from below by

$$
\begin{aligned}
\operatorname{Pr}\left[S_{N}>x, N>x-\varepsilon a(x)\right] & \geq \operatorname{Pr}\left[S_{\lceil x+\varepsilon a(x)\rceil}>x, N>x+\varepsilon a(x)\right] \\
& \geq \operatorname{Pr}[N>x+\varepsilon a(x)]-\operatorname{Pr}\left[S_{\lceil x+\varepsilon a(x)\rceil} \leq x\right] .
\end{aligned}
$$

The second term on the right-hand side is $o(\operatorname{Pr}[N>x])$ as $x \rightarrow \infty$ by the same argument as in the previous paragraph. Moreover, by (2.6),

$$
\lim _{x \rightarrow \infty} \frac{\operatorname{Pr}[N>x+\varepsilon a(x)]}{\operatorname{Pr}[N>x-\varepsilon a(x)]}=e^{-2 \varepsilon} .
$$

As $\varepsilon>0$ was arbitrary, indeed $\operatorname{Pr}\left[S_{N}>x\right] \sim \operatorname{Pr}[N>x]$ as $x \rightarrow \infty$.

Theorem 4.2 If $N$ and $\left\{X_{i}\right\}_{i \geq 1}$ are independent, if $\mathrm{E}\left[e^{\gamma X_{1}}\right]<\infty$ for some $\gamma>0$ and if (2.6) holds for a function a such that

$$
\liminf _{x \rightarrow \infty} \frac{\log a(x)}{\log x}>\frac{1}{2}
$$


then $\operatorname{Pr}\left[S_{N}>x\right] \sim \operatorname{Pr}[N>x / \mu]$ as $x \rightarrow \infty$.

Proof. Without loss of generality, assume $\mu=1$. Let $\lambda(x):=\log a(x) / \log x$, so $a(x)=x^{\lambda(x)}$. By (2.7) and (4.3),

$$
1 / 2<\lambda_{0}:=\liminf _{x \rightarrow \infty} \lambda(x) \leq \limsup _{x \rightarrow \infty} \lambda(x) \leq 1
$$

Let $k$ be an integer larger than 2 such that $\lambda_{0}>1 / 2+1 / 2^{k}$ and decompose

$$
\operatorname{Pr}\left[S_{N}>x\right]=T_{1}(x)+T_{2}(x)+T_{3}(x),
$$

where

$$
\begin{aligned}
& T_{1}(x)=\operatorname{Pr}\left[S_{N}>x, N \leq\left\lfloor x-x^{3 / 4}\right\rfloor\right], \\
& T_{2}(x)=\operatorname{Pr}\left[S_{N}>x,\left\lfloor x-x^{3 / 4}\right\rfloor<N \leq\left\lfloor x-x^{1 / 2+1 / 2^{k}}\right\rfloor\right], \\
& T_{3}(x)=\operatorname{Pr}\left[S_{N}>x,\left\lfloor x-x^{1 / 2+1 / 2^{k}}\right\rfloor<N\right] .
\end{aligned}
$$

We will show that for $i=1,2$,

$$
T_{i}(x)=o(\operatorname{Pr}[N>x]), \quad x \rightarrow \infty
$$

and, for arbitrary $\varepsilon>0$,

$$
e^{-\varepsilon} \leq \liminf _{x \rightarrow \infty} \frac{T_{3}(x)}{\operatorname{Pr}[N>x]} \leq \limsup _{x \rightarrow \infty} \frac{T_{3}(x)}{\operatorname{Pr}[N>x]} \leq e^{\varepsilon}
$$

Since $\varepsilon$ is arbitrary, the combination of equations (4.4), (4.5) and (4.6) implies $\lim _{x \rightarrow \infty} \operatorname{Pr}\left[S_{N}>x\right] / \operatorname{Pr}[N>x]=1$, as required.

The term $T_{1}(x)$.

Denoting $n(x)=\left\lfloor x-x^{3 / 4}\right\rfloor$, we have $T_{1}(x) \leq \operatorname{Pr}\left[S_{n(x)}>x\right]=\operatorname{Pr}\left[S_{n(x)}>\right.$ $n(x)+\{x-n(x)\}]$. Since $x-n(x) \sim x^{3 / 4}$ is of smaller order than $x$ but of larger order than $x^{1 / 2}$ as $x \rightarrow \infty$, an application of Lemma 2.2 yields

$$
T_{1}(x) \leq \exp \left(-\frac{1}{2 \sigma^{2}} x^{1 / 2}\{1+o(1)\}\right), \quad x \rightarrow \infty .
$$

On the other hand, since $x^{1 / 2}=o(a(x))$ as $x \rightarrow \infty$, the representation in (2.8) implies $\exp \left(-\delta x^{1 / 2}\right)=o(\operatorname{Pr}[N>x])$ as $x \rightarrow \infty$ for all $\delta>0$. Equation (4.5) for $i=1$ follows.

The term $T_{2}(x)$. 
For integer $j \geq 2$, write $\tau_{j}=1 / 2+1 / 2^{j}$. Clearly $3 / 4=\tau_{2}>\tau_{3}>\cdots>\tau_{k}>$ $1 / 2$. Further, denote $n_{j}(x)=\left\lfloor x-x^{\tau_{j}}\right\rfloor$. We have $n_{2}(x) \leq n_{3}(x) \leq \cdots$ and

$$
\begin{aligned}
T_{2}(x) & =\operatorname{Pr}\left[S_{N}>x, n_{2}(x)<N \leq n_{k}(x)\right] \\
& =\sum_{j=2}^{k-1} \operatorname{Pr}\left[S_{N}>x, n_{j}(x)<N \leq n_{j+1}(x)\right] \\
& \leq \sum_{j=2}^{k-1} \operatorname{Pr}\left[S_{n_{j+1}(x)}>x\right] \operatorname{Pr}\left[N>n_{j}(x)\right] .
\end{aligned}
$$

A similar argument as for the term $T_{1}(x)$ in the previous paragraph yields

$$
\begin{aligned}
\operatorname{Pr}\left[S_{n_{j+1}(x)}>x\right] & =\exp \left(-\frac{1}{2 \sigma^{2}} x^{2 \tau_{j+1}-1}\{1+o(1)\}\right) \\
& =\exp \left(-\frac{1}{2 \sigma^{2}} x^{1 / 2^{j}}\{1+o(1)\}\right), \quad x \rightarrow \infty .
\end{aligned}
$$

On the other hand, since $x^{1 / 2}=o(a(x))$ as $x \rightarrow \infty$, the representation in $(2.8)$ implies

$$
\log \frac{\operatorname{Pr}\left[N>n_{j}(x)\right]}{\operatorname{Pr}[N>x]}=o\left(x^{1 / 2^{j}}\right), \quad x \rightarrow \infty .
$$

Combine the final two displays to derive that every term on the right-hand side of $(4.7)$ is $o(\operatorname{Pr}[N>x])$ as $x \rightarrow \infty$.

The term $T_{3}(x)$.

Fix $\varepsilon>0$. On the one hand, since $a(x)=x^{\lambda(x)}$, the choice of $k$ entails that $x^{1 / 2+1 / 2^{k}}=o(a(x))$ as $x \rightarrow \infty$. Hence

$$
T_{3}(x)=\operatorname{Pr}\left[S_{N}>x, N>\left\lfloor x-x^{1 / 2+1 / 2^{k}}\right\rfloor\right] \leq \operatorname{Pr}[N>x-\varepsilon a(x)],
$$

whence, by $(2.6), T_{3}(x) \leq\{1+o(1)\} e^{\varepsilon} \operatorname{Pr}[N>x]$ as $x \rightarrow \infty$.

On the other hand, denoting $m(x)=\lfloor x+\varepsilon a(x)\rfloor$,

$$
\begin{aligned}
T_{3}(x) & \geq \operatorname{Pr}\left[S_{N}>x, N>m(x)\right] \\
& \geq \operatorname{Pr}\left[S_{m(x)}>x, N>m(x)\right]=\left\{1-\operatorname{Pr}\left[S_{m(x)} \leq x\right]\right\} \operatorname{Pr}[N>m(x)] .
\end{aligned}
$$

Since $m(x)=x+\{\varepsilon+o(1)\} a(x)$ as $x \rightarrow \infty$, by Lemma 2.2,

$$
\begin{aligned}
\operatorname{Pr}\left[S_{m(x)} \leq x\right] & =\operatorname{Pr}\left[S_{m(x)} \leq m(x)-\{m(x)-x\}\right] \\
& =\exp \left(-\frac{\varepsilon^{2}}{2 \sigma^{2}} \frac{a^{2}(x)}{x}\{1+o(1)\}\right) \rightarrow 0, \quad x \rightarrow \infty
\end{aligned}
$$


Moroever, by (2.6), $\operatorname{Pr}[N>m(x)] \sim e^{-\varepsilon} \operatorname{Pr}[N>x]$ as $x \rightarrow \infty$. This relation in combination with (4.8) and (4.9) yields $T_{3}(x) \geq\{1+o(1)\} e^{-\varepsilon} \operatorname{Pr}[N>x]$ as $x \rightarrow \infty$. This finishes the proof of (4.6) and hence of the theorem.

Example. Let $N$ be a discretized Weibull variable with shape parameter $0<\beta<1$ as in the example in subsection 2.2. Then Theorem 4.1 applies for $\beta<1 / 3$, while Theorem 4.2 applies as long as $\beta<1 / 2$.

\section{Discussion}

At first sight, Theorem 4.2 does not seem to entail much of an extension compared to the following corollary to Theorem 3.6 in Asmussen et al. (1999).

Corollary 5.1 If $N$ and $\left\{X_{i}\right\}_{i \geq 1}$ are independent and if eq. (2.6) holds for a function a such that

(i) $a(n) / n^{1 / 2} \rightarrow \infty$ as $n \rightarrow \infty$,

(ii) $\operatorname{Pr}\left[S_{n}>n \mu+c a(n)\right]=o(\operatorname{Pr}[N \geq n])$ as $n \rightarrow \infty$ for all $c>0$,

then $\operatorname{Pr}\left[S_{N}>x\right] \sim \operatorname{Pr}[N>x / \mu]$ as $x \rightarrow \infty$.

Indeed, the only real difference of Corollary 5.1 with respect to Theorem 4.2 seems to be the extra condition (ii). However, this condition turns out to be not so harmless: Although condition (i) only requires $a(n)$ to grow to infinity at a faster rate than $n^{1 / 2}$, the following lemma shows that condition (ii) effectively forces a much faster rate on $a$, comparable to the one imposed in Theorem 4.1.

Lemma 5.2 Under the conditions of Corollary 5.1, if $\mathrm{E}\left[e^{\gamma X_{1}}\right]<\infty$ for some $\gamma>0$ and if the function a is regularly varying of index $\delta<1$, then necessarily $\delta \geq 2 / 3$.

Proof. By (2.8) and by Lemma 2.2,

$$
\frac{\operatorname{Pr}\left[S_{n}>n \mu+a(n)\right]}{\operatorname{Pr}[N \geq n]}=\exp \left(-\frac{1}{2 \sigma^{2}} \frac{a^{2}(n)}{n}\{1+o(1)\}+\int_{0}^{n} \frac{g(t)}{a(t)} d t-\log c(n)\right)
$$

as $n \rightarrow \infty$. By Karamata's theorem (Bingham et al., 1987, Proposition 1.5.8),

$$
\int_{0}^{n} \frac{g(t)}{a(t)} d t \sim \frac{1}{1-\delta} \frac{n}{a(n)}, \quad n \rightarrow \infty
$$


On the one hand, the function $t \mapsto a^{2}(t) / t$ is regularly varying of index $2 \delta-1$; on the other hand, the function $t \mapsto t / a(t)$ is regularly varying of index $1-\delta$. Hence, if the expression in (5.1) converges to zero as $n \rightarrow \infty$, then necessarily $2 \delta-1 \geq 1-\delta$, whence $\delta \geq 2 / 3$.

Finally, the lower bound $1 / 2$ for the order of the mean excess function $a$ in Theorem 4.2 seems to mark the minimal weight that must be present in the tail of $N$ for the asymptotic equivalence $\operatorname{Pr}\left[S_{N}>x\right] \sim \operatorname{Pr}[N>x / \mu]$ to be true. Assume for example that the distribution of $X_{i}$ is unit-mean exponential and that $N$ is independent of $\left\{X_{i}\right\}_{i \geq 1}$. For $t \geq 0$, the distribution of the random variable $Z_{t}=\max \left\{n=0,1, \ldots: S_{n} \leq t\right\}$ is Poisson with mean $t$, and

$$
\operatorname{Pr}\left[S_{N}>t\right]=\operatorname{Pr}\left[N>Z_{t}\right]=\mathrm{E}\left[\exp \left\{-g\left(Z_{t}\right)\right\}\right]
$$

where $g(x):=-\log \operatorname{Pr}[N>x]$. The asymptotic behavior of the final expression in the previous display as $t \rightarrow \infty$ has been studied in Foss and Korshunov (2000) in the general case where $Z_{t}$ is the sum of iid nonnegative random variables; in our case, $Z_{t}$ is for integer $t$ the sum of independent random variables with common Poisson distribution and mean 1. By Foss and Korshunov (2000, Theorem 5.1), if $N=\lfloor Y\rfloor$ and $\operatorname{Pr}[Y>y]=\exp \left(-y^{\beta}\right)$ for $y \geq 0$ where $\beta \in[1 / 2,2 / 3)$, then

$$
\operatorname{Pr}\left[S_{N}>x\right] \sim \operatorname{Pr}[N>x] \exp \left(\beta^{2} x^{2 \beta-1} / 2\right), \quad x \rightarrow \infty .
$$

In particular, $\operatorname{Pr}\left[S_{N}>x\right]$ is not asymptotically equivalent to $\operatorname{Pr}[N>x]$. In Asmussen et al. (1999), the exponent $1 / 2$ was found to be critical as well.

\section{Applications}

Earthquake insurance. Earthquake insurance provides coverage to the policyholder in the event of an earthquake that causes damage to the policyholder's properties. Insurance companies must be careful when assigning this type of insurance because, even if the individual claims are bounded by the value of the properties insured, the number of claims can be very large. An earthquake strong enough to destroy one house will probably destroy hundreds of houses in the same area.

Let us assume that, given the energy of the earthquake $\Lambda=\lambda$, the number of claims has a Poisson distribution with parameter $\beta \lambda$ where $\beta$ is a positive constant. Despite of the apparent complexity involved in the dynamics of earthquakes, the probability distribution of the energy of an earthquake follows a power law distribution known as the Gutenberg-Richter law (Gutenberg and Richter, 1944): $\operatorname{Pr}[\Lambda>\lambda]=\lambda^{-\alpha} L(\lambda)$ where the exponent $\alpha$ is an 
universal exponent close to 1 , universal in the sense that it does not depend on a particular geographic area, and $L$ is a slowly varying function.

We claim that as $x \rightarrow \infty$,

$$
\operatorname{Pr}[N>x] \sim \operatorname{Pr}[\Lambda>x / \beta]=(x / \beta)^{-\alpha} L(x / \beta) .
$$

The proof goes as follows. Without loss of generality, assume $\beta=1$. Let $F_{\lambda}$ denote the distribution function of the Poisson distribution with mean $\lambda$. Note that if $0<\lambda<\mu<\infty$, then $1-F_{\lambda}<1-F_{\mu}=1-F_{\lambda} * F_{\mu-\lambda}$. Also let $Z_{1}, Z_{2}, \ldots$ denote iid random variables with common Poisson distribution and mean 1 . Then

$$
\begin{aligned}
\operatorname{Pr}[N>x] & =\mathrm{E}[\operatorname{Pr}(N>x \mid \Lambda)]=\mathrm{E}\left[1-F_{\Lambda}(x)\right] \\
& \leq \mathrm{E}\left[1-F_{\lceil\Lambda\rceil}(x)\right] \\
& =\operatorname{Pr}\left[\sum_{i=1}^{\lceil\Lambda\rceil} Z_{i}>x\right]
\end{aligned}
$$

and similarly

$$
\operatorname{Pr}[N>x] \geq \operatorname{Pr}\left[\sum_{i=1}^{\lfloor\Lambda\rfloor} Z_{i}>x\right] .
$$

Since the tail function of $\Lambda$ is regularly varying and since $\Lambda-1<\lfloor\Lambda\rfloor \leq\lceil\Lambda\rceil<$ $\Lambda+1$, we find as $\lambda \rightarrow \infty$

$$
\operatorname{Pr}[\lfloor\Lambda\rfloor>\lambda] \sim \operatorname{Pr}[\Lambda>\lambda] \sim \operatorname{Pr}[\lceil\Lambda\rceil>\lambda] .
$$

The claim now follows from our Theorem 3.1 and the above upper and lower bounds on $\operatorname{Pr}[N>x]$.

By Theorem 3.1, the distribution of the total claim amount in the portfolio has a regularly varying tail as well. Let us note that it is not necessary to assume that the individual claim amounts are independent of the claim number. In fact the energy of the earthquake may also have an impact on the distribution of the individual claim amounts.

Hierarchical unobserved heterogeneity. Let us consider an hierarchical heterogeneity model for the number, $N$, of claims of a policyholder. First assume that, given $\Lambda=\lambda$, the claim number $N$ has a Poisson distribution with parameter $\lambda$. Secondly assume that, given $V=v, \Lambda$ has an exponential distribution with mean $v>0$. It follows that

$$
\operatorname{Pr}[N=n \mid V=v]=\frac{v}{1+v} \frac{1}{(1+v)^{n}},
$$


that is, given $V=v, N$ follows a Geometric distribution with succes probability parameter $v /(1+v)$. Thirdly assume that $\log (1+V)$ follows a Gamma distribution with shape parameter $\gamma>0$ and scale parameter $c>0$. We deduce that

$$
\operatorname{Pr}[N \geq n]=\mathrm{E}\left[e^{-n \log (1+V)}\right]=\left(\frac{c}{c+n}\right)^{\gamma},
$$

that is, $N$ has a Pareto distribution with index $\gamma$.

It is well-known that the omission of an individual unobserved heterogeneity leads to overdispersion (in the sense that the variance is larger with heterogeneity). The proposed hierarchical model shows that unobserved heterogeneity can lead to grossly incorrect conclusions about the tail of the claim number distribution. Assume that their is no heterogeneity for the claim distributions and that the common distribution is light-tailed. If the heterogeneity for the number of claims is observed, then the distribution of the total claim amount is light-tailed, whereas if the heterogeneity is unobserved, then the distribution of the total claim amount is heavy-tailed.

Stationary waiting time of customers. Let $\left\{T_{i}\right\}_{i \geq 1}$ be a stationary sequence of nonnegative random variables with finite mean and set $S_{n}=$ $\sum_{i=1}^{n} T_{i}$. In Resnick and Samorodnitsky (1997), the number of customers, $N_{n}$, in a system seen by the $n$th arriving customer is defined by $N_{0}=0$ and

$$
N_{n}=\left(N_{n-1}+1-\Gamma\left(S_{n-1}, S_{n}\right)\right)_{+}, \quad n \geq 1
$$

where $\Gamma$ is a homogeneous Poisson process with intensity $\mu$ independent of $\left\{T_{i}\right\}_{i \geq 1}$. The waiting time, $W_{n}$, of the $n$th arriving customer satisfies

$$
W_{n} \stackrel{d}{=} \sum_{i=1}^{N_{n}+1} X_{i}
$$

where $X_{1}, X_{2}, \ldots$ are iid exponentially distributed random variables with common mean $\mu$ independent of $N_{n}$. If $\left\{T_{i}\right\}_{i \geq 1}$ is a reversible, stationary, ergodic process and if $\mathrm{E}\left[T_{1}\right]<\mu$, then $N_{n}$ converges in distribution to a random variable $N$, the distribution of which may, under additional conditions, have a regularly varying tail. Whereas in Resnick and Samorodnitsky (1997) only a lower bound is given for the tail of

$$
W \stackrel{d}{=} \sum_{i=1}^{N+1} X_{i}
$$

Theorems 3.1 and 3.2 give sufficient conditions such that actually

$$
\operatorname{Pr}[W>x] \sim \operatorname{Pr}\left[N>\mu^{-1} x\right], \quad x \rightarrow \infty .
$$


Teletraffic arrivals. A large number of teletraffic measurements shows that file sizes and transmission times exhibit heavy tails and long-range dependence. Standard models for explaining these empirically observed facts are the so-called ON/OFF model and the infinite source Poisson model. In Fay et al. (2006), a model is introduced that extends these standard models in a simple, but realistic way. They assume that the first packet of a flow of data arrives at the point $\Gamma_{j}$ of a Poisson process with intensity $\lambda>0$. Flow $j$ then consists of $K_{j}$ packets, the $k$ th of which arrives at time $Y_{j k}=\Gamma_{j}+S_{j k}$, where

$$
S_{j k}=\sum_{i=1}^{k} X_{j i}, \quad 0 \leq k \leq K_{j} .
$$

In Fay et al. (2006), it is assumed that the variables $X_{j i}$ form an array of iid nonnegative random variables and the $K_{j}$ are iid integer-valued random variables independent of the $X_{j i}$. Of interest is the tail behavior of the total transmission time $S_{j K_{j}}$ under the assumption that the tail of $X_{j i}$ or $K_{j}$ is regularly varying. Proposition 4.3 of Fay et al. (2006) gives results similar as those in Theorem 3.2 above. In Fay et al. (2006, Proposition 4.9), the reverse problem is considered as well: if the tail of $S_{j K_{j}}$ is regularly varying with index $-\alpha$ for some $\alpha>0$ and if the tail of $K_{j}$ is heavier than that of $X_{j i}$, then what can be said about $K_{j}$ ? For instance, if the tail of $S_{j K_{j}}$ is regularly varying with index $-\alpha \neq-1$ and if $\operatorname{Pr}\left[X_{1}>x\right]=o(\operatorname{Pr}[N>x])$ as $x \rightarrow \infty$, then the tail of $K_{j}$ must be regularly varying with index $-\alpha$ as well.

\section{References}

Anderson, C. W., 1980. Local limit theorems for the maxima of discrete random variables. Mathematical Proceedings of the Cambridge Philosophical Society 88, 161-166.

Asmussen, S., Klüppelberg C., Sigman K., 1999. Sampling at subexponential times, with queueing applications. Stochastic Processes and their Applications 79, 265-286.

Baltrunas, A., Daley, D.J., Klüppelberg, C., 2004. Tail behaviour of the busy period of a GI/G/1 queue with subexponential service times. Stochastic Processes and their Applications 111, 237-258.

Beard, R., Pentikainen, R., Pesonen, E., 1984. Risk Theory. Chapman \& Hall, Boca Raton.

Bingham, N. C., Goldie, C. M., Teugels, J. L., 1987. Regular Variation. Cambridge University Press, Cambridge.

Cai, J., Tang, Q., 2004. On max-sum equivalence and convolution closure of heavy-tailed distributions and their applications. Journal of Applied Probability 41 (1), 117-130.

Cline, D. B. H., 1994. Intermediate regular and $\Pi$ variation. Proceedings of the London Mathematical Society, Third series 68 (3), 594-616. 
Cline, D. B. H., Samorodnitsky, G., 1994. Subexpontiality of the product of independent random variables. Stochastic Processes and their Applications 49, 75-98.

Denisov, D., Foss, S., Korshunov, D., 2007. On lower limits and equivalences for distribution tails of randomly stopped sums. EURANDOM Report 2007004, http://www.eurandom.nl/.

Embrechts, P., Klüppelberg, C., Mikosch, T., 1997. Modelling Extremal Events for Finance and Insurance. Springer-Verlag, Berlin.

Fay, G., Gonzalez-Arevalo, B., Mikosch, T., Samorodnitsky, G., 2006. Modeling teletraffic arrivals by a Poisson cluster process. Queueing Systems 54 (2), 121-140.

Feller, W., 1971. An Introduction to Probability Theory and Its Applications, Volume II. John Wiley \& Sons, New York.

Foss, S., Korshunov, D.A., 2000. Sampling at a random time with a heavytailed distribution. Markov Processes and Related Fields 6, 643-658.

Gutenberg, R. and Richter, C.F., 1944. Frequency of earthquakes in California. Bulletin of the Seismological Society of America 34, 185-188.

Jensen, J.L., 1995. Saddlepoint Approximations. Oxford University Press.

Kaas, R., Goovaerts, M., Dhaene, J., Denuit, M., 2001. Modern Actuarial Risk Theory. Kluwer Academic Publishers, Boston.

Kaas, R., Tang, Q., 2005. A large deviations result for aggregate claims with dependent claim occurrences. Insurance: Mathematics and Economics 36 (3), 251-259.

Ng, K.W., Tang, Q., Yan, J., Yang, H., 2004. Precise large deviations for sums of random variables with consistently varying tails. Journal of Applied Probability 41 (1), 93-107.

Resnick, S., Samorodnitsky, G., 1997. Performance decay in a single server exponential queueing model with long-range dependence. Operations Research 45 (2), 235-243.

Schmidli, H., 1999. Compound sums and subexponentiality. Bernoulli 5, 999 1012.

Stam, A. J., 1973. Regular variation of the tail of a subordinated distribution. Advances in Applied Probability 5, 308-327.

Tang, Q., 2004. Asymptotics for the finite time ruin probability in the renewal model with consistent variation. Stochastic Models 20 (3), 281-297.

Tang, Q., 2006. Insensitivity to negative dependence of the asymptotic behavior of precise large deviations. Electronic Journal of Probability 11, 107-120. 